\title{
Dramatic course of unusual remote complication of surgical aorta coarctation repair treated with endovascular methods
}

\author{
Krzysztof Pyra ${ }^{1}$, Maciej Szmygin ${ }^{1}$, Michał Sojka $^{1}$, Kamil Baczewski², Tomasz Jargiełło ${ }^{1}$ \\ ${ }^{1}$ Department of Interventional Radiology and Neuroradiology, Medical University of Lublin, Lublin, Poland \\ ${ }^{2}$ Department of Cardiosurgery, Medical University of Lublin, Lublin, Poland
}

Adv Interv Cardiol 2020; 16, 2 (60): 224-226

DOI: https://doi.org/10.5114/aic.2020.96071

Coarctation of the aorta $(\mathrm{COA})$ is a discrete narrowing in the aortic arch causing obstruction to the flow of blood. Untreated CoA carries a poor prognosis with average survival between 30 and 40 years of age and $75 \%$ mortality by 46 years of age [1]. The types of surgical repair include: resection with end-to-end anastomosis with or without graft interposition, patch aortoplasty, bypass procedure or subclavian flap aortoplasty. At present, no single treatment modality appears to have a clear superiority. Since the late 1970s authors have reported remote complications of the repair including aneurysm and pseudo-aneurysms formation, aortic recoarctation (ReCoA) or dissection. Studies showed a considerable increase of late morbidity among patients who underwent this procedure [2, 3].

The authors present a dramatic course of unusual aortic damage in a patient with history of surgical repair of CoA over 40 years prior to hospitalization treated with endovascular methods.

A 55-year-old woman was admitted to the Emergency Unit of a district hospital from a wedding complaining of weakness, lower limb paresthesia (she fell while dancing) and abdominal discomfort. The patient reported a history of surgical treatment (lack of medical documentation) due to coarctation of the aorta at the age of 8 years and percutaneous closure of atrial septal defect (ASD) with an Amplatzer Septal Occluder 1 year prior to the current hospitalization. After several hours in the Emergency Unit of a district hospital where she reported the severity of existing complaints and the appearance of new symptoms in the form of abdominal pain and tachypnea she was referred to the Cardiosurgery Department. On admission: tachypnea, paraparesis, abdominal pain, hypoactive bowel sounds and non-palpable peripheral pulse.
Blood count revealed critical values of coagulation tests (international normalized ratio (INR) 4.7), signs of renal dysfunction (creatinine - $1.1 \mathrm{mg} / \mathrm{dl}$, estimated glomerular filtration rate (eGFR) - $51 \mathrm{ml} / \mathrm{min} / 1.73 \mathrm{~m}^{2}$ ) and anemia (hemoglobin (HGB) $10.1 \mathrm{~g} / \mathrm{dl}$, red blood cells (RBC) $3.9 \times 10^{6} / \mathrm{ml}$ ). Computed tomography (CT)-angio revealed aortic stenosis below the level of the operated region and probably presence of a thrombus (Figures $1 \mathrm{~A}-\mathrm{H}$ ). The patient was qualified for thoracic stent graft implantation in order to restore aortic flow and cover the thrombus to prevent its migration.

Under all sterile conditions and in general anesthesia the right femoral artery was punctured and a vascular sheath introduced. The aortic narrowing was passed with a soft hydrophilic wire (TERUMO, MA, USA) over which a pigtail catheter was advanced. Initial angiography confirmed critical stenosis of the aorta at the end of the prostheses, the shape of which was visible in digital subtraction angiography (DSA) (Figures $1 \mathrm{l}, \mathrm{J})$ ). Afterwards, the soft guidewire was replaced by an Amplatz Guidewire (Cook Medical, IN, USA) which due its stiffness changed its position compared to the previous guide wire. From the marginal part of the aorta it moved inward, which could suggest damage to the thrombus. During preparation for the stent graft implantation restoration of pulse on the femoral artery was noticed. Then, a long $6 \mathrm{Fr}$ vascular sheath was introduced above the stenosis and control angiography performed. It depicted significant improvement in blood flow due to the stiff Amplatz guidewire which moved as it turned out the "vascular flap". This flap, located below the distal prosthesis suturing, acted like a sail which closed the aortic lumen (Figure $1 \mathrm{~K})$. After that the treatment plan was modified. An Epic self-expanding stent (Boston Scientific, MA, USA)

\section{Corresponding author:}

Dr. Maciej Szmygin, Department of Interventional Radiology and Neuroradiology, Medical University of Lublin, Lublin, Poland e-mail: mszmygin@gmail.com

Received: 13.01.2020, accepted: 29.01.2020. 

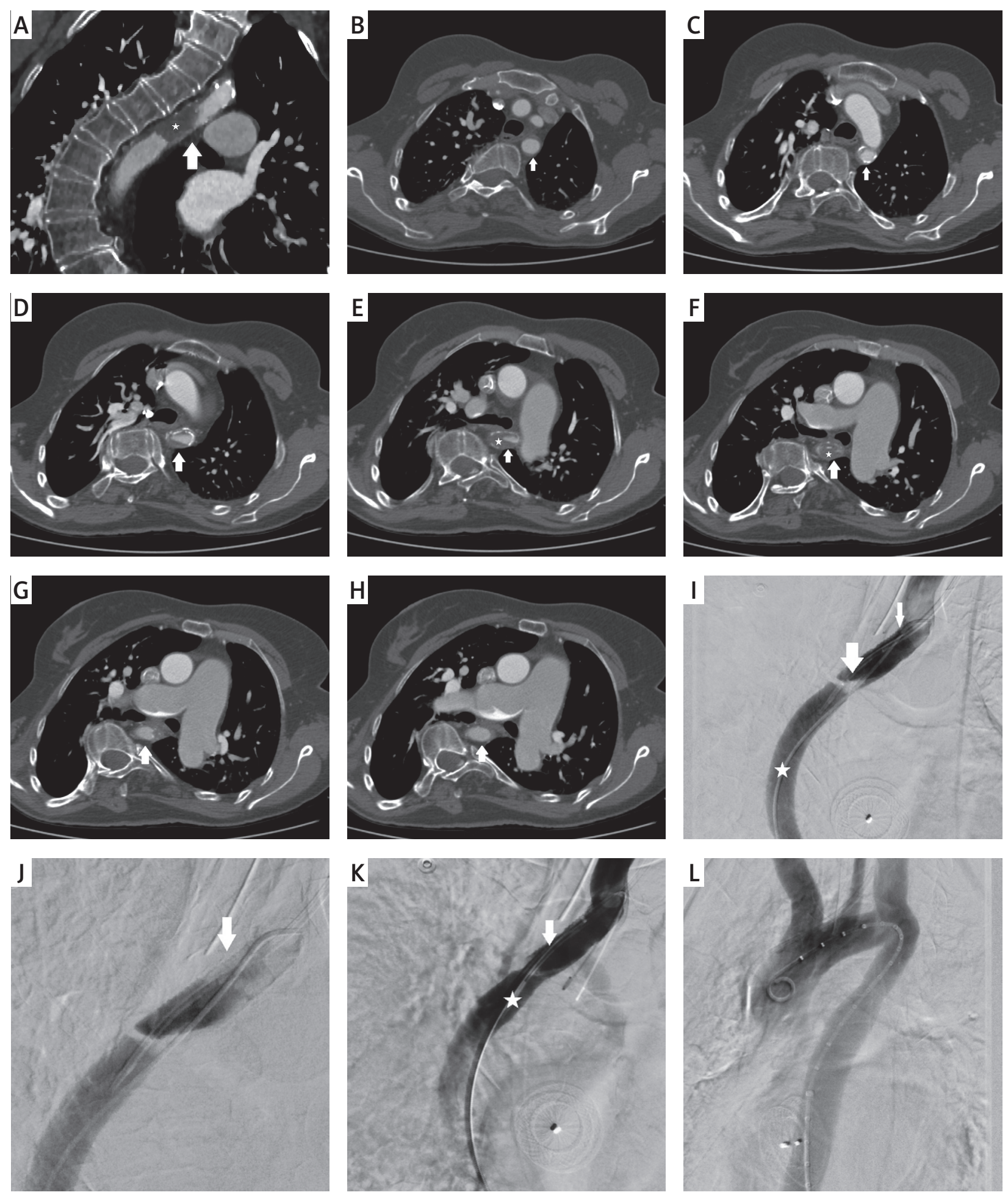

Figure 1. A - Coronal angio-CT with visible narrow lumen of the vessel (arrow) with HU corresponding to the density of the thrombus (star). B-H - Further transverse CT scans showing critical stenosis of the aorta caused most likely by the presence of the thrombus (stars). I - Initial angiography after introduction of the $5 \mathrm{Fr}$ catheter (star) over the soft guidewire. Visible critically stenosed lumen with contrast-filled "pocket" on the opposite side. Thin arrow - proximal end of the prosthesis. Thick arrow - central line. J - Enlargement of the stenosed area with visible irregular shape of the prosthesis (arrow). $\mathbf{K}$ - Control angiography after introduction of the $6 \mathrm{Fr}$ (star) over the stiff guidewire. Arrow shows free flap offset caused by the stiff guidewire and vascular sheath leading to partial restoration of the blood flow. L - Final angiography after stent implantation disclosing proper patency of the vessel and full restoration of the blood flow 
was introduced. There was no need for postdilatation. The stent fully expanded (Figure $1 \mathrm{~L}$ ). Final aortography revealed proper stent placement and adequate perfusion of the infra-aortic vessels. Peripheral pulse was restored. Visceral and lower limb artery angiography was performed. Narrow vessels were visible, and flow was properly maintained.

After the procedure the clinical condition of the patient started to deteriorate due to reperfusion injury. Symptoms of renal and circulatory insufficiency were observed (INR - 6.2, creatinine - $1.8 \mathrm{mg} / \mathrm{dl}$, eGFR - $31 \mathrm{ml}$ $\mathrm{min} / 1.73 \mathrm{~m}^{2}$ ) and renal replacement therapy introduced. Critical blood test results confirmed suspicion of gastrointestinal bleeding ( $\mathrm{HGB}-4.0 \mathrm{~g} / \mathrm{dl}, \mathrm{RBC}-1.3 \times 10^{6} / \mathrm{ml}$ ). Despite adequate medication and renal replacement therapy the patient died $7 \mathrm{~h}$ after surgery.

In our opinion, the damage occurred as a result of increased physical exertion and increased pressure, which resulted in damage to the intima at the site of the distal prosthesis suturing. The created intimate flap acted like a sail. It was pushed by the blood flow to the opposite wall of the aorta, thus closing the flow

The angio CT image in correlation with the angiographic image is still not fully understood. In angio CT a contrast loss is visible, which has no equivalent in angiography. The CT was examined by two experienced radiologists on a calibrated radiological monitor. Densities in Hounsfield units corresponded to the density of the thrombus. The thrombus can be located inside the lumen of aorta or it can be a thrombosed false lumen. However, no intimal flap was present on the CTA and the image was not typical for dissection. The next option, where no sign of an intimal tear is observed, which could have been taken into consideration in differential diagnosis, was hemorrhage into the wall from the vasa vasorum. Nonetheless, intramural hematomas do not narrow the lumen so much. Hence the most likely CT diagnosis was a thrombus.

If it had been in fact a thrombus that migrated before or during the procedure, we would have had a large vessel occlusion. In the final angiography we could see that the vessels were properly patent. It was possible that the damage was dynamic and the flap moved, which resulted in creating "pockets". It is certain that this was a piece of intima, and that the radial force of the self-expendable stent flattened it against the wall of the aorta. In time, noticeable pulse restoration helped to avoid thoracic stent graft implantation, which in this patient with an extremely narrow aorta would be significantly too oversized. The safest and best solution would be implantation of a covered stent graft of the right size, unfortunately not available at that time. The situation required urgent intervention, especially as the patient had lost several hours of valuable time before.
In conclusion, early diagnosis and treatment is crucial for patients with such findings. Patients with symptoms of lower extremities ischemia, especially with a history of cardiac surgery, should therefore receive special attention in order to prevent dramatic events. The described case proves that the interpretation of CTA images is not always clear and might differ from the DSA images.

\section{Conflict of interest}

The authors declare no conflict of interest.

\section{References}

1. Warnes CA, Williams RG, Bashore TM, et al.; ACC/AHA 2008 Guidelines for the Management of Adults With Congenital Heart Disease: A Report of the American College of Cardiology/ American Heart Association Task Force on Practice Guidelines (Writing Committee to Develop Guidelines on the Management of Adults With Congenital Heart Disease). Circulation 2008; 118: e714-833.

2. Toro-Salazar $\mathrm{OH}$, Steinberger J, Thomas $\mathrm{W}$, et al. Long-term follow-up of patients after coarctation of the aorta repair. Am J Cardiol 2002; 89: 541-7.

3. Maron BJ, Humphries JO, Rowe RD, Mellits ED. Prognosis of surgically corrected coarctation of the aorta. A 20-year postoperative appraisal. Circulation 1973; 47: 119-26. 